\title{
Ilhas de calor urbano em Montes Claros - MG
}

\section{Urban heat island in Montes Claros - MG}

\author{
Maria Ivete Soares de Almeida* $₫(\mathbb{D}$, Lucas Augusto Pereira da Silva $\bowtie(\mathbb{D}$, \\ Marcos Esdras Leite $₫(1)$, Carla Milena de Moura Laurentino $\square$ (I), \\ Samuel Carlos Santos Marques $₫(\mathbb{D})$, Manoel Reinaldo Leite $\varangle$ (D)
}

\author{
Departamento de Geociências, Universidade Estadual de Montes Claros, Montes Claros, Minas \\ Gerais, Brasil \\ E-mail: lucaskaio1605@gmail.com (LAPS) ; marcosesdrasleite@gmail.com (MEL) ; \\ carlamilenaml@gmail.com (CMML); Samuel35marques@gmail.com (SCSM); \\ leitemanoelreinaldo@gmail.com (MRL) \\ *E-mail para correspondência: ivetegeo@yahoo.com.br
}

Recebido (Received): 31/10/2019 Aceito (Accepted): 10/12/2020

Resumo: O objetivo desse trabalho é analisar a distribuição espacial das Ilhas de Calor na Cidade de Montes Claros - MG. A escolha dessa área se justifica pelo crescimento populacional e a expansão urbana que a mesma vem passando nos últimos anos. Para execução desse trabalho, dotou do uso de técnicas de sensoriamento remoto para modelagem térmica e índices de comprimentos de onda. Foram utilizados os produtos orbitais dos satélites Landsat 5 e 8 (sensores TM e OLI/TIRS, respectivamente) referentes às datas: 28/08/1985 e 18/09/2016. Os resultados mostraram que a distribuição espacial das Ilhas de Calor está relacionada as alterações na ocupação e uso do solo urbano, variando em escala espaço-temporal, apresentando intensificação nas regiões centrais para as anomalias positivas, e nas bordas apresentam aumento das distribuições de anomalias negativas, sendo essas correlatas ao incremento de áreas verdes em condomínios de alto padrão. A elevada de temperatura de superfície possui relação proporcional com o NDBI e inversamente com o NDVI, considerando as características da cobertura do solo. Esse estudo se mostra como instrumento de planejamento urbano, sobretudo considerando os aspectos de uso e ocupação do solo para com efeitos no âmbito meteorológico e climatológico, haja vista que estes elementos são essenciais para o bem estar humano.

Palavras-Chaves: Temperatura; NDBI; NDVI; Sensoriamento Remoto.

Abstract: The objective of this work is to analyze the spatial distribution of the Heat Islands in the city of Montes Claros - MG. The choice of this area is justified by the population growth and the urban expansion that it has been going through in recent years. To carry out this work, it provided the use of remote sensing techniques for thermal modeling and wavelength indices. Orbital products from the Landsat 5 and 8 satellites (TM and OLI / TIRS sensors, respectively) were used for the dates: 08/28/1985 and 09/18/2016. The results showed that the spatial distribution of the Heat Islands is related to changes in the occupation and use of urban land, varying in spatiotemporal scale, intensifying in the central regions for positive anomalies, and in the edges presenting an increase in negative anomaly distributions. These are correlated with the increase of green areas in high standard condominiums. The elevated surface temperature temperature is proportionally related to NDBI and inversely to NDVI, considering the characteristics of the ground cover. This study is shown as an urban planning instrument, especially considering the aspects of land use and occupation for meteorological and climatic effects, considering that these elements are essential for human well-being.

Keywords: Temperature; NDBI; NDVI; Remote Sensing. 


\section{Introdução}

O crescimento do espaço urbano tende a desencadear uma série de processos degradantes ao ambiente, elevando os riscos naturais, que vão repercutir direta e indiretamente no bem estar social. Este crescimento no Brasil se deu, sobretudo em função das elevadas taxas de urbanização e concentração de pessoas que as cidades brasileiras sofreram a partir da década de 1950 (COMITRE; ANDRADE, 2011). Nessa perspectiva, dados secundários apontaram que até 2010 mais de $84 \%$ da população brasileira e $50 \%$ da população mundial residiam em áreas urbanas (TRINDADE et al., 2017).

Este fato contribui para as pressões exercidas sobre os recursos naturais, principalmente no que diz respeito às mudanças do uso e ocupação do solo urbano. Essas alterações, têm se mostrado como indicadores responsáveis por distorções na circulação atmosférica (dinâmica de ventos locais), lançamento de particulados e alteração nos padrões climáticos de tais áreas (BARROS; LOMBARDO, 2016).

Diante disso, salienta-se que as cidades produzem seus próprios climas, sobretudo em função das variações no balanço de radiação e energia, e, diante essas, tem-se o surgimento do fenômeno Ilha de Calor (BARROS; LOMBARDO, 2016). Este fenômeno representa as ilhas de Calor (em dimensão horizontal e vertical) em ambientes urbanos resultantes da absorção e armazenamento diferencial de calor pelos materiais nesses espaços; no âmbito científico são reconhecidos três tipos de ilhas de calor urbano: de atmosfera inferior, superior e de superfície.

O aumento da temperatura, em função das ilhas de calor urbano, altera o fluxo de materiais e de energia nos sistemas ecológicos urbanos. Isso fomenta modificações sistemáticas em: questões hidrológicas urbanas, propriedades pedológicas, dinâmica atmosférica, hábitos biológicos, ciclos de matéria e material, metabolismo energético e saúde daqueles que residem nesses ambientes (SOLTANI; SHARIFI, 2017).

Alguns estudos realizaram abordagens com intuito de analisar as ilhas de calor urbano dispondo de instrumental que compreendem áreas pontuais (WOO, 2016; SANTOS et al., 2017). Entretanto, com o advento do desenvolvimento tecnológico, o sensoriamento remoto orbital aparece como ferramenta ímpar para este fim, notadamente considerando as diferentes resoluções espaciais e temporais fornecidas pelos sensores orbitais. Diante disso, o sensoriamento remoto tem sido amplamente utilizado em análises meteorológicas e climatológicas com intuito de compreender as nuances das ilhas de calor (CORRÊA et al., 2016; TRINDADE et al., 2017).

Dessa forma, o objetivo desse trabalho é analisar a distribuição espacial das ilhas de calor urbano na Cidade de Montes Claros/MG. A escolha dessa cidade se deve ao aumento populacional dessa cidade a partir da década de 1970, bem como sua expansão territorial nos últimos anos (LEITE et al., 2012; LEITE et al., 2014). Esses processos provocaram alterações no uso e ocupação do solo urbano, o que aumentou a área impermeável e edificada na periferia da cidade, além de aumentar a densidade das edificações no interior do perímetro urbano.

\section{Materiais e métodos}

\subsection{Localização da área de estudo}

O município de Montes Claros está localizado na Mesorregião Norte de Minas Gerais (Figura 1), possui área de $3568.941 \mathrm{~km}^{2}$ (IBGE, 2010). No censo de 2010, a população de Montes Claros era 361.915 habitantes (IBGE, 2010). Entretanto, as estimativas apontaram o município com 404 habitantes, em 2018 (IBGE, 2018). A população urbana, em 2010, representava 95,17\% (344.427 habitantes) do total para este período, e a rural, com 4,83\% (17.488 habitantes) (IBGE, 2010). No que diz respeito ao Índice de Desenvolvimento Humano Municipal (IDHM), em escala temporal, houve aumentos significativos, sendo: 0,514, 0,661 e 0,770, em 1991, 2000 e 2010, respectivamente (FJP, 2010).

Montes Claros possui clima tropical quente semiúmido, com verão quente e inverno seco, com precipitação média de $1082,4 \mathrm{~mm}$, com temperatura média anual de $23,5{ }^{\circ} \mathrm{C}$, com máxima e mínima pontuadas em $25,05{ }^{\circ} \mathrm{C}$ e $20,5{ }^{\circ} \mathrm{C}$ (LEITE, 2011). No contexto Geológico, a área é marcada pela bacia sedimentar Neoproterozóica do São Francisco e rochas siliciclásticas Mezopoterozóicas da Serra do Espinhaço, na borda Sudeste do Cráton do São Francisco (LEITE, 2011). As unidades geológicas predominantes no município são: Cobertura Superficial Indiferenciada, Grupo Urucuia, Formação Lagoa do Jacaré, Formação Serra da Saudade e Serra de Santa Helena (CODEMIG, 2015).

Do ponto de vista geomorfológico, Montes Claros possui: planícies fluviais $(<550 \mathrm{~m})$, superfície de aplainamento $(<650 \mathrm{~m})$, planaltos de níveis intermediários $(<759 \mathrm{~m})$, planaltos dissecados $(<900 \mathrm{~m})$, 
planalto tabular $(<1000 \mathrm{~m})$ e chapadões de topo de cimeira $(>1000 \mathrm{~m})$ (LEITE; BRITO, 2012). A pedologia é centrada nos seguintes tipos de solos: latossolos vermelho-amarelo, cambissolo háplico, e litossolo háplico (DPS/UFV, 2010). A vegetação do município de Montes é classificada como Cerrado stricto sensu, floresta decidual e semidecidual. A área de estudo é a cidade de Montes Claros, que se caracteriza por possuir adensamento elevado devido ao processo de expansão urbana (LEITE, 2010). Do ponto de vista econômico, menciona-se o destaque às prestações de serviços (LEITE, 2010). Tangente ao setor educacional, a cidade apresenta-se bem desenvolvida, com universidades públicas - Universidade Estadual de Montes Claros (UNIMONTES), Instituto de Ciências Agrárias (ICA-UFMG), Instituto Federal do Norte de Minas Gerais (IFNMG) - e faculdades privadas, sendo essenciais para o desenvolvimento de regiões adjacentes.

O estudo foi realizado considerando as regiões de planejamento (Figura 1), estabelecidas pela Prefeitura Municipal (decreto $\mathrm{n}^{\circ} 3.393$ ), as quais possuem como objetivo o planejamento estratégico, sobretudo a gestão de políticas públicas e o contexto de desenvolvimento econômico.
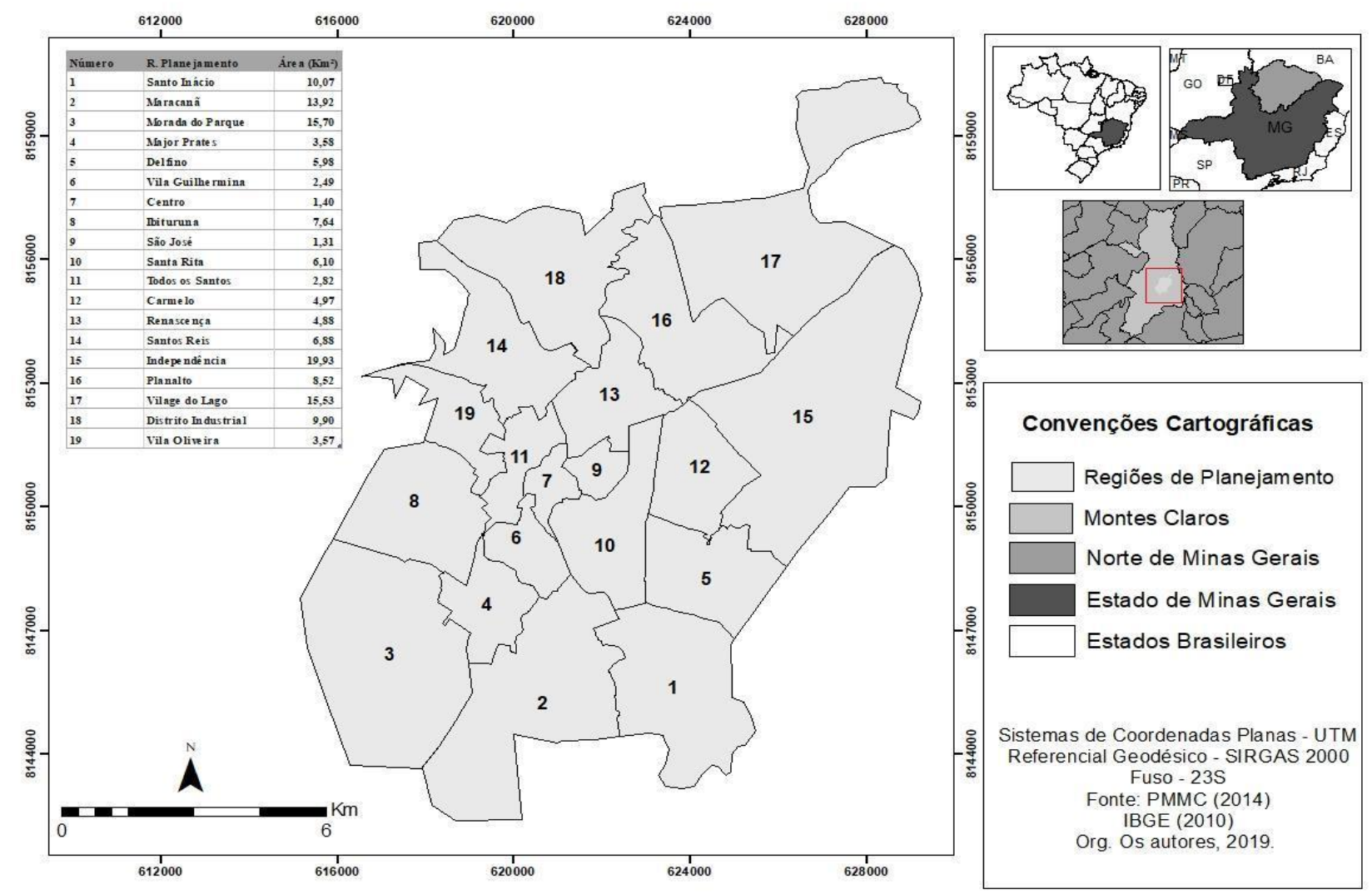

Figura 1: Localização da área de estudo.

De acordo com a Figura 1, foram estabelecidas 19 regiões de planejamento na cidade de Montes Claros: Santo Inácio, Maracanã, Morada do Parque, Major Prates, Delfino, Vila Guilhermina, Centro, São José, Santa Rita, Todos os Santos, Carmelo, Renascença, Santos Reis, Independência, Planalto, Vilage do Lago, Distrito Industrial e Vila Oliveira.

\subsection{Procedimentos técnicos-operacionais}

Para execução deste trabalho foram utilizados produtos orbitais disponibilizados pelo Serviço Geológico dos Estados Unidos - USGS. Os produtos compreendem os períodos de agosto de 1985 e setembro de 2016. Esses produtos são provenientes dos satélites Landsat 5 e 8 (sensores TM e OLI/TIRS, respectivamente). Sendo obtidas para a órbita e ponto: 219/072.

Essas imagens são de período seco (julho e agosto) - do ponto de vista da sazonalidade climática. Essa escolha se deu pelo fato de não haver nuvens durante a passagem do sensor, as quais poderiam inferir nos resultados, sobretudo devido às distorções na radiância nos diferentes comprimentos de onda. Outro motivo para essa escolha, é que os primeiros dados para o Landsat 5 em disposições de período seco, foram no ano de 1985. Os produtos cartográficos no formato shapefile para as regiões de planejamento foram fornecidos pela Prefeitura Municipal de Montes Claros (PMMC). 
Para o processamento dos dados, foram utilizados os softwares Erdas e ArcGis 10.5. No Erdas, foram gerados os índices de comprimento de onda (NDVI - Índice de Vegetação pela Diferença Normalizada NDBI - Índice Acumulado pela Diferença Normalizada) e Temperatura de Superfície (Ts). No ArcGis plataforma Arcmap - foram feitos os layouts, bem como conversão da Ts da escala Kelvin para Graus Centígrados e análise de Ilhas de Calor. No que diz respeito à espacialização dos dados demográficos, foram utilizadas bases oficiais do IBGE do censo 2010; e para análise de densidade residencial, utilizou-se documentos cartográficos fornecidos pela Prefeitura Municipal de Montes Claros. Na Tabela 1 estão dispostas as equações dos índices espectrais, Ts, obtenção das Ilhas de Calor, bem como as bandas utilizadas:

Tabela 1 - Descrições dos Cálculos realizados.

\begin{tabular}{|c|c|}
\hline $\begin{array}{l}\text { Bandas: } 1,2,3,4,5,6 \text { e } 7 \\
\text { para Landsat }-5\end{array}$ & $\begin{array}{l}0.45-0.52,0.50-0.60,0.63-0.69,0.76-0.90,1.55-1.75,10.4-12.5,2.08-2.35 \text {, } \\
\text { micrometros, respectivamente. }\end{array}$ \\
\hline $\begin{array}{l}\text { Bandas: } 2,3,4,5,6,7 \text { e } 10 \\
\text { para Landsat }-8\end{array}$ & $\begin{array}{l}0.45-0.51,0.53-0.59,0.64-0.67,0.85-0.88,1.57-1.65,2.11-2.29,10.60- \\
11.19, \text { micrometros, respectivamente. }\end{array}$ \\
\hline $\begin{array}{l}\text { Índice de Vegetação pela Diferença } \\
\text { Normalizada - NDVI }\end{array}$ & $\frac{I v p-\operatorname{Red}}{I v p+\operatorname{Red}}$ \\
\hline $\begin{array}{l}\text { Índice Construtivo pela Diferença } \\
\text { Normalizada - NDBI }\end{array}$ & $\frac{I v m-I v p}{I v m+I v p}$ \\
\hline Temperatura de Superfície & $\mathrm{T}_{\mathrm{s}}=\frac{\mathrm{K}_{2}}{\ln \left(\frac{\varepsilon_{\mathrm{NB}} \mathrm{K}_{1}}{\mathrm{~L}_{\lambda, 6}}+1\right)}$ \\
\hline $\begin{array}{l}\text { Conversão de Kelvin para } \\
\text { Centígrados. }\end{array}$ & $\begin{array}{l}\text { Para Landsat } 5 \text { utilizou-se a banda } 6 \text { e para Landsat } 8 \text {, a banda } 10 \text {. } \\
\text { Conversão: Ts }-273.15\end{array}$ \\
\hline Ilhas de Calor Urbano & $\mathrm{Ts}^{\circ} \mathrm{C}-\mathrm{Média} \mathrm{Ts}^{\circ} \mathrm{C}$ \\
\hline
\end{tabular}

Fonte: Rouse et al., (1973); Tucker (1979); Zha et al., (2003); Trindade et al., (2017).

O NDVI sendo a razão dos comprimentos de onda representativos do infravermelho próximo (Ivp) e vermelho visível $(R e d)$ - dado pelo espalhamento intracelular das folhas da vegetação, nos espaços vazios de ar, da radiação eletromagnética e a absorção desta pela clorofila presente nas folhas, respectivamente varia de -1 a 1 , e é utilizado para analisar mudanças no uso e cobertura da terra e vegetação urbana.

O NDBI, formulando-se a partir dos comprimentos de onda infravermelho médio (Ivm) e infravermelho próximo (Ivp), varia de -1 a 1 , tende a corresponder com valores elevados àqueles alvos que há menor absorção da radiação e os menores valores são destinados a áreas com maior absorção a radiação por água, nessa perspectiva, é utilizado para análises em áreas urbanas, identificando, sobretudo edifícios e solos em exposição (GUHA et al., 2018).

A temperatura de superfície (Ts) foi obtida utilizando a radiância espectral no canal emissivo (banda termal), bem como a emissividade termal - que segue os fundamentos físicos de Planck, em que a capacidade do corpo de emitir radiação, varia conforme sua estrutura atômica, sendo a razão entre a emitância do corpo real e a máxima (corpo negro) - advinda do índice de área foliar (IAF) - que representa a cobertura do solo pelo dossel vegetativo (razão da área do dossel e da área projetada no solo) (GOMES et al., 2017). Os dados são obtidos na escala térmica de Kelvin (K), portanto, foi inerente ao estudo a conversão para graus centígrados.

As Ilhas de Calor Urbano (ICU) foram obtidas tendo a diferença entre as médias térmicas de cada pixel do limite das regiões de planejamento em relação à média geral deste limite (TRINDADE et al., 2017) extraída pela estatística na plataforma ArcMap do ArcGis. Com isso, assume-se que os valores de Ts acima 
ou abaixo da média geral, podem representar anomalias no campo térmico, configurando ilhas de calor ou frescor em ambientes urbanos.

\section{Resultados e discussão}

Inicialmente será analisada a distribuição espacial da Ts, NDVI e NDBI para os dois períodos (1985 e 2018), buscando compreender o comportamento dessas variáveis no âmbito da área urbana de Montes Claros. Em seguida, serão discutidas as Ilhas de Calor.

\subsection{Distribuição espacial de Ts, NDVI e NDBI para 1985 e 2018}

Para o período analisado em 1985 , a Ts teve média estimada em $29{ }^{\circ} \mathrm{C}$, com valores mínimo e máximo em $36,9{ }^{\circ} \mathrm{C}$ e $21,5{ }^{\circ} \mathrm{C}$, respectivamente, tendo desvio padrão médio calculado em $1,37{ }^{\circ} \mathrm{C}$, a amplitude térmica foi de $15,4^{\circ} \mathrm{C}$. No que diz respeito ao NDVI, os valores médio, máximo e mínimo foram de: 0,35 , $0,83,-0,69$, respectivamente, tendo desvio padrão de 0,19 . Os valores médio, máximo e mínimo para NDBI foram na ordem de 0,11, 0,53 e -0,52, respectivamente, e desvio padrão calculado em 0,1 (Figura 2):

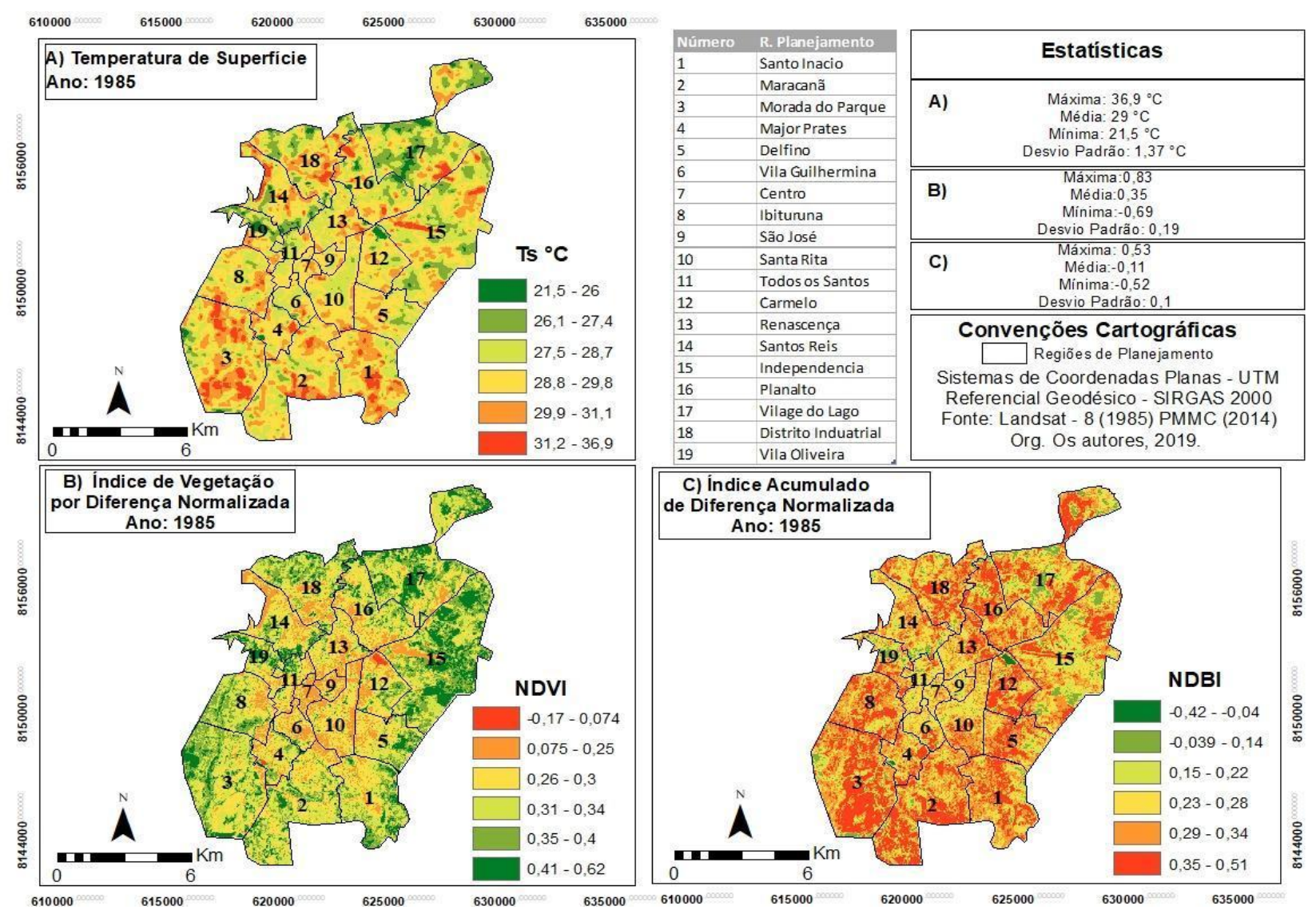

Figura 2: Ts, NDVI e NDBI para 1985.

Para 2016, os valores médio, máximo e mínimo de Ts foram estimados em $31,34{ }^{\circ} \mathrm{C}, 37,61{ }^{\circ} \mathrm{C}$ e 22,86 ${ }^{\circ} \mathrm{C}$, respectivamente. O desvio padrão foi calculado na ordem de $1,57^{\circ} \mathrm{C}$, e a amplitude foi de $14,76{ }^{\circ} \mathrm{C}$. Em outros estudos, foram encontrados valores de Ts para áreas urbanas entre $14^{\circ} \mathrm{C}$ e $49{ }^{\circ} \mathrm{C}$ (BARBOSA; VECCHIA, 2007; OLTRA-CARRIÓ et al., 2010; STEINKE et al., 2010; CORRÊA et al., 2012; COELHO; CORREA, 2013; NEVES et al., 2018). O NDVI apresentou média de 0,24, valores máximo e mínimo de 0,83 e $-0,69$, respectivamente, com desvio padrão estimado em 0,19. Estudos mostram que o NDVI para diferentes usos do solo em ambientes urbanos pode variar entre -0,02 e 0,69 (LOBATO et al., 2010; GUILHERME et al., 2016; BARBOSA et al., 2017). O NDBI para 2016 obteve valores médio, máximo e mínimo de 0,15, 0,48 e -0,15, respectivamente (Figura 3), o NDBI variou em outros trabalhos na ordem de 0,02 e 1 (SAKUNO et al., 2017; XU et al., 2018). 

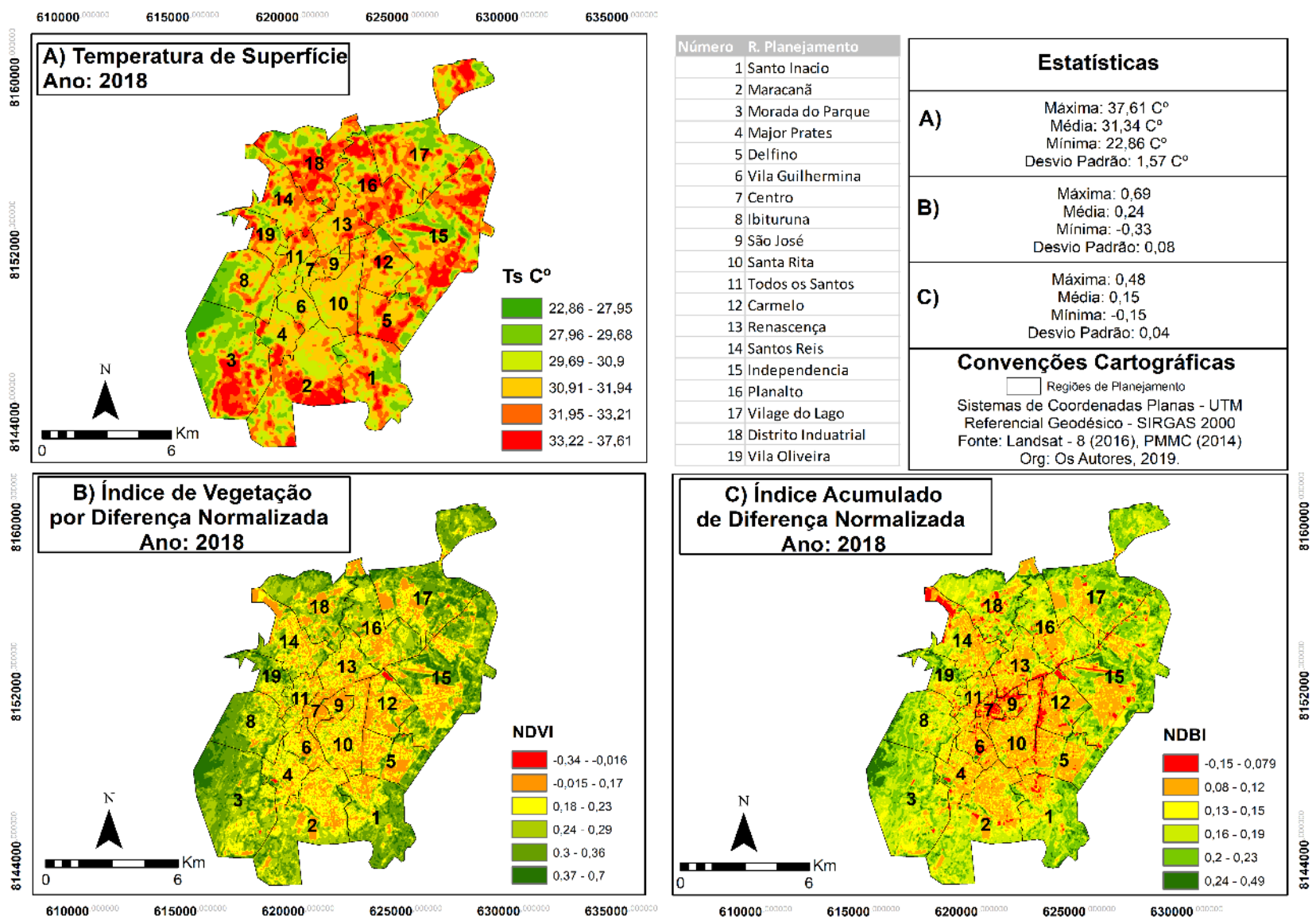

Figura 3: Ts, NDVI e NDBI para 2016.

Analisando em conjunto os dois períodos (1985 e 2016) observa-se variação significativa nos 3 elementos analisados (Ts, NDVI e NDBI). No mapa B da Figura 2, observa-se que há maior distribuição dos valores de NDVI entre 0,35 e 0,62 havendo concentração no norte e no nordeste (nas regiões de planejamento Vilage do Lago e Independência).

Em 2016, observa-se uma concentração dos valores entre 0,30 e 0,70 a sudoeste, destacando nas regiões do Ibituruna e do Morada do Parque. Em análises para a mesma área de estudo, foi observado o mesmo comportamento para essas regiões de planejamento (PEREIRA et al., 2017).

Na Figura 4 estão dispostas as cartas imagens, focando nas regiões de planejamento Morada do Parque e Ibiturura, tanto em 1985, quanto em 2016, para explicitar a concentração dos valores de NDVI.

É perceptível que há predominância de vegetação no ano de 2016 em relação a 1985, estando subdividido entre Cerrado stricto sensu, Floresta Estacional Decidual e Mata Ciliar. Isso fomenta o acréscimo do espalhamento de radiação no espectro infravermelho próximo e absorção no vermelho visível - NDVI (HUETE et al., 2002), bem como decréscimos significativos do NDBI, devido a absorção do canal do infravermelho médio, em função do conteúdo de água presente na vegetação (MARQUES, 2013).

A Ts para as regiões de planejamento Morada do Parque e Ibituruna, apresentou leve acréscimo de 1985 para 2016, antes (1985) a concentração dos valores estava entre $27,5^{\circ} \mathrm{C}$ e $36,9^{\circ} \mathrm{C}$, já em 2016 predominam as classes entre 27,96 e $37,61{ }^{\circ} \mathrm{C}$. No período de 1985 observa-se que as classes com menores médias termais apresentam valores entre $21,5^{\circ} \mathrm{C}$ e $26^{\circ} \mathrm{C}$. Em 2016, para essas classes, foram estimados valores na ordem de 22,86 e $27,95{ }^{\circ} \mathrm{C}$. Os valores elevados para 2016 estão relacionados às concepções da física, no contexto da segunda lei da termodinâmica, tendo em vista que os corpos mais quentes transferem calor para os corpos mais frios. 

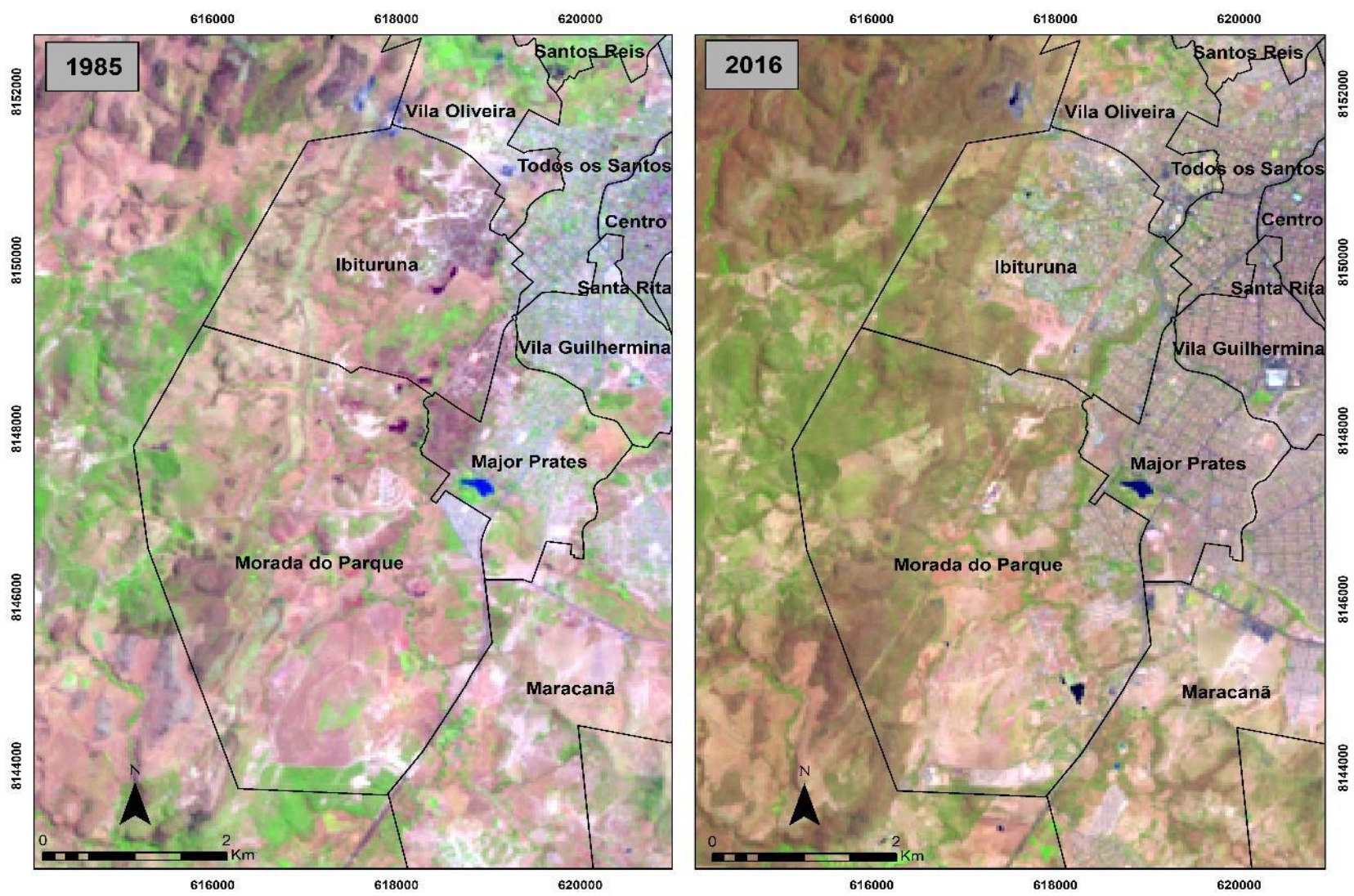

Figura 4: Cartas das imagens em RGB.

Cabe ressaltar que análises realizadas nas mesmas latitudes apresentaram que alterações no campo térmico de usos da terra implicam em equilíbrio termodinâmico no mesmo segmento encontrado para esse estudo (LEITE, 2011). É possível observar que há maior distribuição espacial dos valores com médias mais elevadas em 2016, principalmente nas regiões de planejamento Maracanã, Major Prates, Delfino, Vila Guilhermina, São José, Santa Rita, Renascença, Santos Reis, Independência, Planalto, Vilage do Lago e Distrito Industrial, já em 1985 foram observadas maiores distribuições para Santo Inácio, Maracanã, Morada do Parque, Major Prates e Independência.

A observação dos valores elevados, bem como dos mais amenos no campo térmico, são essenciais para a fundamentação das discussões a respeito das ICU - no próximo tópico - principalmente diante dos métodos assumidos neste estudo.

\subsection{Ilhas de calor urbano}

As ICU estão bem distribuídas em ambos os períodos. Para este trabalho, os valores que variaram $3^{\circ} \mathrm{C}$ acima da média foram apontados como ilhas de calor. Observa-se que ao sul da área de estudo tem-se concentrações de valores situados nas classes $5,30{ }^{\circ} \mathrm{C}$ e $7,86{ }^{\circ} \mathrm{C}$, sobretudo no extremo sul da região de planejamento (RP) Morada do Parque e em porções do Maracanã, bem como na RP do Santo Inácio. Em 1985, a distribuição espacial das ilhas de calor (positivas) apresenta-se relativamente mais espaçadas, (Figura 5).

Chama-se atenção para a pista do aeroporto RP Independência, tanto em 1985, quanto em 2016, esse tipo de ocupação está inserido nas classes de anomalias positivas mais elevadas. Estando associado à estrutura física da área, contendo materiais em que a admitância térmica é elevada, sendo responsável pelo acréscimo nas médias termais.

Nas regiões de planejamento do Vilage do Lago, Independência, Maracanã (ao norte), Major Prates, Santos Reis, Planalto, Distrito Industrial, Delfino (a sudeste) - no contexto de 1985 e 2016 - a temperatura de superfície elevada (e consequentemente as ICU) se justifica pela intensa concentração de construções fomentado pelo elevado número de moradores e de novas construções (Figura 6), além disso essas regiões possuem baixo valor de terra e encontram-se em uma distância considerável do centro, o que influenciou no processo de ocupação. 


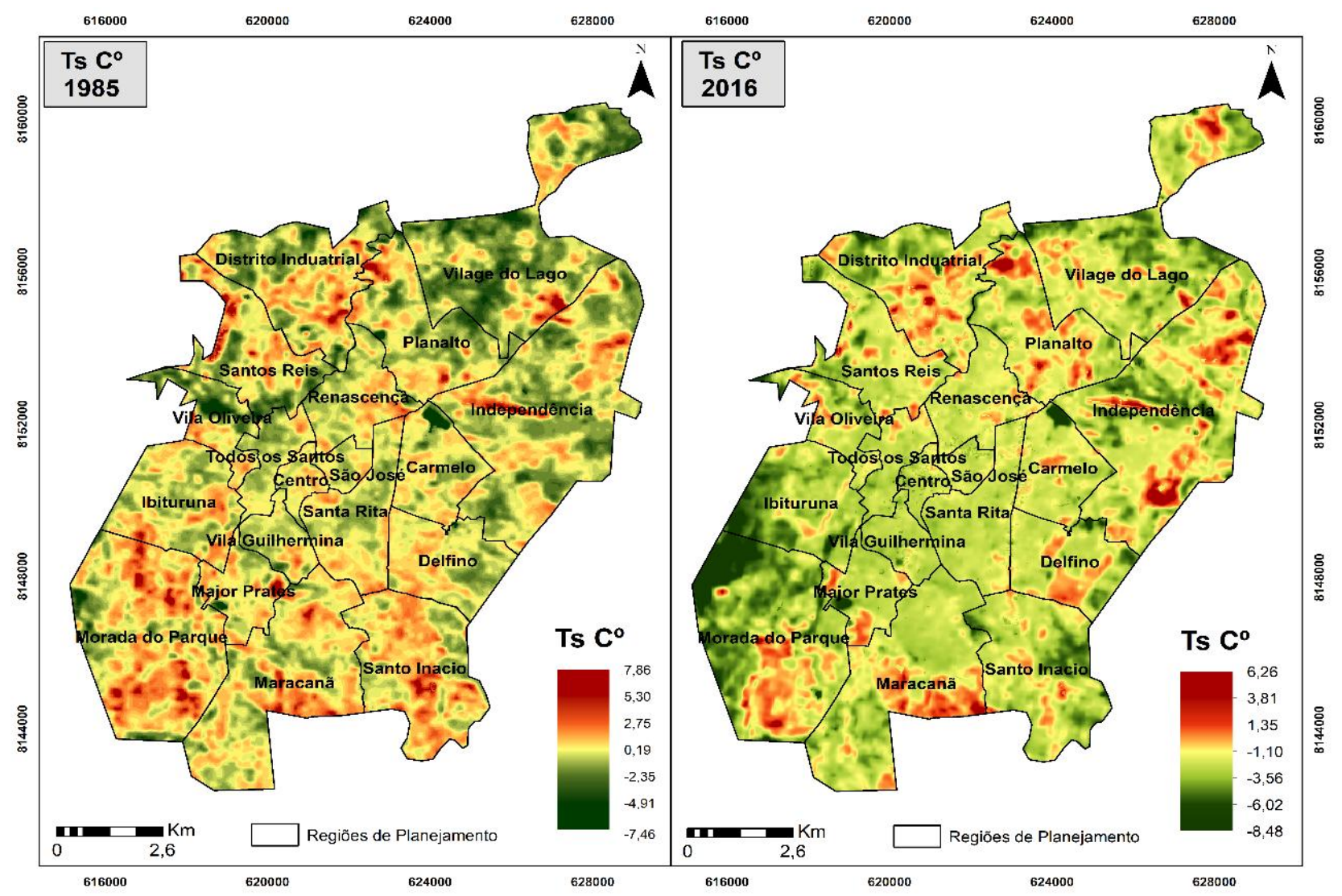

Figura 5: Distribuição Espacial das Ilhas de Calor. Fonte: Landsat 5 e 8, 1985 e 2018.

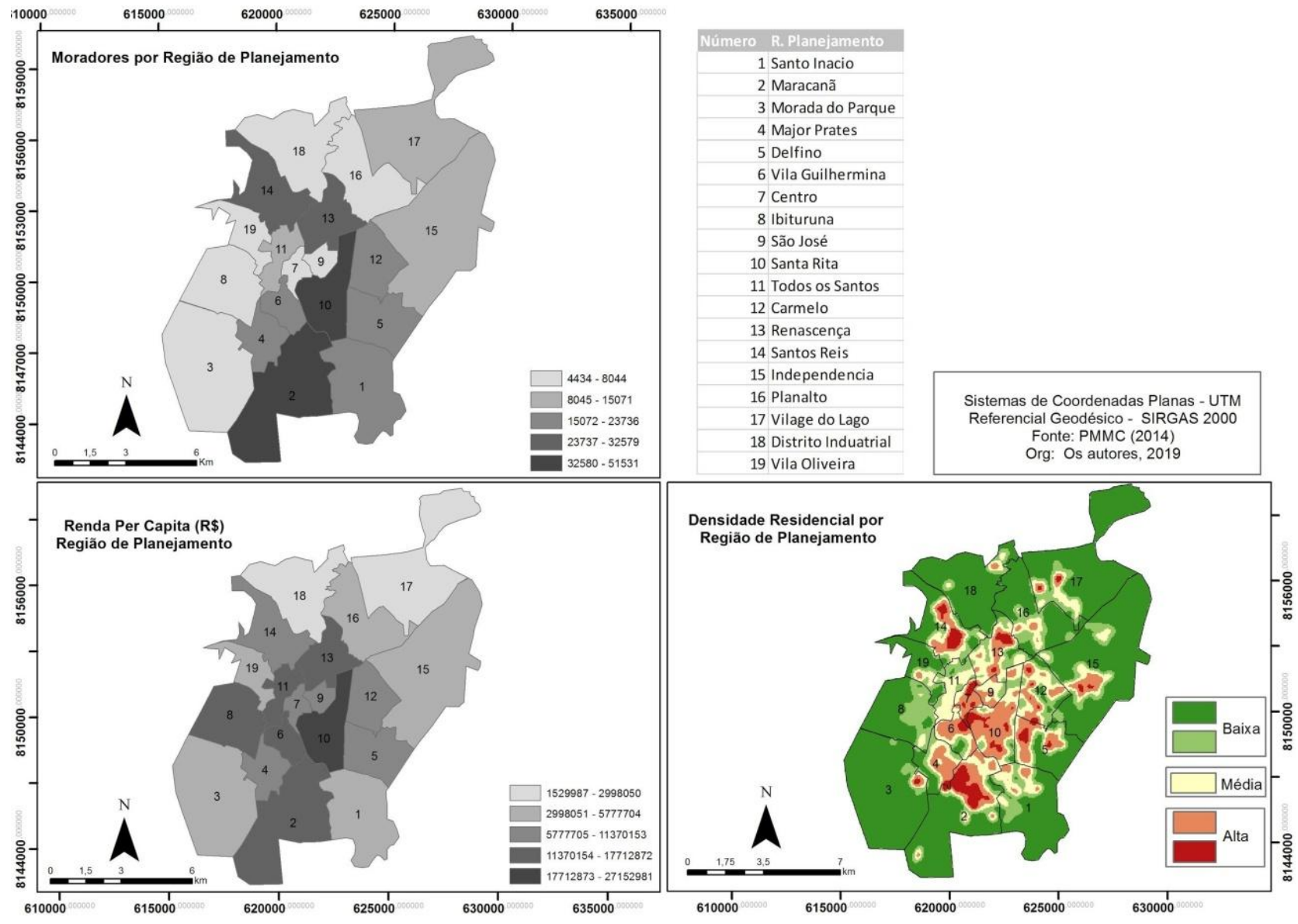

Figura 5: Moradores, Renda Per Capita, Densidade Residencial das Regiões de Planejamento 
Em função do baixo valor da terra, a população que se concentrou nessa região possui a renda per capita relativamente baixa (SILVA; TRAVASSOS, 2008; FEITOSA et al., 2011; LEITE; BRITO; 2012) - asfalto, e com escassez de áreas verdes, o que contribui para o desequilíbrio térmico. Para explicitar essa intensificação e aumento da distribuição espacial das ICU positivas, menciona-se o adensamento construtivo que Montes Claros, bem como a configuração de uso do solo, visto que, é marcado por: áreas edificadas, árvores, grama, solo exposto, solo britado, calçadas, pavimentos, asfaltos e águas (lagos e rios) (GOMES, 2008). Em um contexto mais generalizado, pode-se pontuar as seguintes classes: residencial, comercial e serviços, industrial, parques e áreas não ocupadas (LEITE et al., 2014). Salienta-se que, o adensamento urbano é justificativa em alguns estudos que analisaram os fenômenos de ilha de calor (TRINDADE et al., 2017).

Em contrapartida, na região sudoeste da cidade, especificamente na região de planejamento do Ibituruna, encontra-se o preço de terra mais elevado de Montes Claros (IBGE, 2010), onde se tem a maior presença dos condomínios horizontais. O Ibituruna apresenta baixa densidade demográfica, 5746 moradores, o que equivale a aproximadamente $1,7 \%$ do total populacional da cidade, ocupando uma área de 7,64\% do perímetro urbano (Figura 6).

Em virtude do elevado preço da terra e abrigar população de alta renda, há áreas não ocupadas, em que existe cobertura vegetal natural, além de constar áreas verdes dentro dos condomínios. O elevado valor da terra e a estrutura das construções da região de planejamento do Ibituruna representa o alto padrão de vida dos moradores que remete à uma renda per capita entre $\mathrm{R} \$ 1625,91$ a $\mathrm{R}$ \$ 2445,40 (LEITE, 2010; FRANÇA, 2015).

É perceptível que há incremento na distribuição dos valores de ICU negativas - comparando 1985 com 2016 - (Figura 5). Este fato é observado principalmente nas bordas das regiões de planejamento. Ainda nessa comparação 1985 - 2016, é inerente apontar que nas áreas centrais há um decréscimo sistemático nas anomalias negativas. A distribuição das ICU negativas, estão associadas aos condomínios de alto padrão, bem como as áreas verdes inseridas nos mesmos; outros estudos apontam que os condomínios de alto padrão tendem a decrescer o campo térmico e o NDBI, e aumentar os índices de vegetação.

Áreas de vegetação tendem a usar maiores porções de radiação solar para o processo de evapotranspiração, umedecendo o ambiente, consequentemente, decrescendo as médias termais, mostrandose como importantes reguladores térmicos.

Além dos aspectos vegetacionais, é oportuno mencionar a influência de materiais adequados, loteamentos extensos, na contribuição do decréscimo da Ts e consequentemente das ilhas de calor, visto que essas condições favorecem a circulação do ar (GOMES et al., 2017).

Há relação intrínseca entre os aspectos demográficos apontados neste trabalho com as Ilhas de Calor, principalmente quando observa-se que em áreas com maior adensamento construtivo e número de moradores, bem como menor renda, houve predominância de Ilhas de Calor, cenário inverso ocorre em regiões com menor adensamento e número de moradores, além de apresentar população com maior renda.

\section{Considerações finais}

Diante das análises expostas, para as regiões de planejamento de Montes Claros foram identificadas intensas variações dentre os índices de comprimento de onda (NDBI e NDVI) e temperatura de superfície.

Essas variações ocorreram diante da lógica espaço temporal (1985 a 2016), sobretudo considerando as mudanças nos aspectos de uso do solo urbano de Montes Claros. As variáveis utilizadas possuem sinergia explícita, apresentando-se como excelentes ferramentas para análises urbanas.

As ilhas de calor urbano apresentaram distribuições sistemáticas na cidade de Montes Claros. As anomalias positivas foram intensificadas nas áreas centrais no intervalo 1985-2016 - em função da intensa concentração de construções - e, as anomalias negativas apresentaram padrões de distribuição nas bordas adjacentes às centrais (principalmente Ibituruna e Morada do Parque), indicando decréscimo no campo térmico em função da ação antrópica - sendo associada ao alto padrão dos condomínios, bem como a baixa densidade construtiva.

Nessa perspectiva, esse estudo se mostra como instrumento de planejamento urbano, sobretudo considerando os aspectos de uso e ocupação do solo para com efeitos no âmbito climatológico e meteorológico, haja vista que estes elementos são essenciais para o bem estar humano. 


\section{Referências}

BARBOSA, A. H. da S.; CARVALHO, R. G.; CAMACHO, R. G. V. Aplicação do NDVI para a Análise da Distribuição Espacial da Cobertura Vegetal na Região Serrana de Martins e Portalegre-Estado do Rio Grande do Norte. Revista do Departamento de Geografia, v. 33, p. 128-143, 2017. https://doi.org/10.11606/rdg.v33i0.128171

BARBOSA, R. V. R.; VECCHIA, F. A. S. Estudos de ilha de calor urbana por meio de imagens do Landsat 7 ETM+: estudo de caso em São Carlos (SP). Revista Minerva, v. 6, n. 3, p. 273-278, 2009.

BARROS, H. R.; LOMBARDO, M. A. A ilha de calor urbana e o uso e cobertura do solo no município de São Paulo-SP. GEOUSP: Espaço e Tempo (Online), v. 20, n. 1, p. 160-177, 2016. https://doi.org/10.11606/issn.2179-0892.geousp.2016.97783

BRASIL, Atlas do Desenvolvimento Humano do Brasil. Disponível em: <http://www.atlasbrasil.org.br/> . 2010.

CODEMIG, COMPANHIA DE DESENVOLVIMENTO ECONÔMICO DE MINAS GERAIS. Mapa Geológico de Minas Gerais. Disponível em: <http://www.portalgeologia.com.br/index.php/mapa/>. 2019.

COELHO, A. L. N.; CORREA, W. de S. C.; NASCIMENTO, F. H. Uso de produtos de sensoriamento remoto para a classificação e análise de temperaturas de superfície no município de Vila Velha/ES (Brasil). GeoFocus. Revista Internacional de Ciencia y Tecnología de la Información Geográfica, v. 1, n. 13, p. 61-76, 2013.

COMITRE, F.; ANDRADE. Crescimento Populacional e Contradições no Espaço Urbano. Uma Análise Da Expansão Periférica Na Cidade Média De Sorocaba-Sp. In: II Simpósio de Cidades Médias e Pequenas da Bahia, 2011, Vitória da Conquista. Anais... Vitória da Conquista: UESB, 2011. v. 1. p. 1-15.

CORRÊA, P. B.; CORRÊA, J. A. de J.; ANDRADE, Silvia C. de P. Análise da temperatura de superfície da área urbana de Santarém através de imagens termais do Landsat 5. Revista Geonorte, v. 2, n. 4, p. 714-722, 2012.

CORRÊA, P. Batista; CANDIDO, L. A.; SOUZA, R. A. F.; ANDREOLI, R. V.; KAYANO, M. T. Estudo do Fenômeno da Ilha de Calor na Cidade de Manaus/AM: Um Estudo a Partir de Dados de Sensoriamento Remoto, Modelagem e Estações Meteorológicas. Revista Brasileira de Meteorologia, v. 31, n. 2, p. 167176, 2016. http://dx.doi.org/10.1590/0102-778631220150012

FRANÇA, I. S.; ALMEIDA, M. I. S.; SILVA, F. G. Cidades médias, fluxos aéreos: o papel regional de Montes Claros/MG. Caderno Prudentino de Geografia, v. 1, n. 37, p. 62-71, 2015.

GOMES, W.; AMORIM, M.; DUBREUIL, V. Modelagem da Ilha de Calor Urbana Aplicada ao Ambiente Litorâneo - Ubatuba/Brasil. Revista do Departamento de Geografia, v. 34, p. 82-94, 22 dez. 2017. https://doi.org/10.11606/rdg.v34i0.133193

GOMES, L. C. F.; SANTOS, C. A. C.; HERMES, A. A. Balanço de Energia à Superfície para a Cidade de Patos-PB Usando Técnicas de Sensoriamento Remoto. Revista Brasileira de Geografia Física. v.6, n.1, p.15-28, 2013. https://doi.org/10.11606/rdg.v34i0.133193

GOMES, P. S.; LAMBERTS, R. O estudo do clima urbano e legislação urbanística: considerações a partir do caso Montes Claros-MG. Ambiente Construído, v. 9, n. 1, p. 73-91, 2009.

GUHA, S.; HIMANSHU, G.; DEY A.; GILL, N. Analytical study of land surface temperature with NDVI and NDBI using Landsat 8 OLI and TIRS data in Florence and Naples city, Italy. European Journal of Remote Sensing, v. 51, n. 1, p. 667-678, 2018. https://doi.org/10.1080/22797254.2018.1474494

HUETE, A.R., DIDAN, K., MIURA, T., RODRIGUEZ, E. P.; GAO, X.; FERREIRA, L.G. Overview of the radiometric and biophysical performance of the MODIS vegetation indices. Remote Sensing of Environment, v. 83, n. 1, p. 195-213, 2012. https://doi.org/10.1016/S0034-4257(02)00096-2 
IBGE, INSTITUTO BRASILEIRO DE GEOGRAFIA E ESTATÍSTICA. Disponível em: <https://www.ibge.gov.br/>. 2010.

LEIZ; OPPENHEIMER, M.; ZHU, Q.; BALDWIN, J. W.; EBI K. L.; BOU-ZEID, E.; XU, L. et al. Interactions between urban heat islands and heat waves. Environmental research letters, v. 13, n. 3, p. 034003, 2018.

LEITE, M. E.; BATISTA, R. P.; CLEMENTE, C. M. S. Segregação espontânea na cidade de Montes Claros/MG: Uma análise auxiliada pelo Sensoriamento Remoto. Geoambiente On-line, n. 15, p. 01-25, 2010. https://doi.org/10.5216/revgeoamb.v0i15.26012

LEITE, M. E.; BRITO, J. L. S. Sensoriamento Remoto e SIG na identificação de áreas propensas à formação de favelas: o caso de Montes Claros (MG). Boletim Goiano de Geografia, v. 32, n. 2, p. 159-175, 2012.

LEITE, M. E.; BRITO, J. L. Sensoriamento remoto aplicado ao mapeamento do uso do solo urbano e de assentamentos ilegais em Montes Claros/MG. Geosul, v. 26, n. 52, p. 99-128, 2011. https://doi.org/10.5007/2177-5230.2011v26n52p99

LEITE, M. E.; PEREIRA, A. M.; NOBRE, B. A.; MARTIN, A. S. Monitoramento da dinâmica no uso do solo urbano de Montes Claros. Caminhos de Geografia, v. 15, n. 51 p. 172-180, 2014.

LEITE, M. R. Sensoriamento remoto aplicado na análise temporal da relação uso da terra/temperatura e albedo de superfície na bacia do Rio Vieira no norte de Minas Gerais. Uberlândia, MG. 2011. Dissertação (Mestrado em Geografia). Uberlândia: UFU, 2011.

LEITE, M. R.; BRITO, J. L. S. Avaliação de desempenho do algoritmo SEBAL na modelagem térmica da bacia do rio Vieira no norte de Minas Gerais. Revista Brasileira de Cartografia, n. 64, v.5, p. 161-174, 2013.

LOBATO, R.; MENEZES. J.; LIMA, L. A.; SAPIENZA, J. A. Índice de vegetação por diferença normalizada para análise da redução da mata atlântica na região costeira do distrito de Tamoios-Cabo Frio/RJ. Caderno de Estudos Geoambientais-CADEGEO, v.01, n.01, p.14-22, 2010.

MARQUES, J. M.; GRAÇA, H.; EGGENKAMP, H. G. M.; NEVES, O.; CARREIRA, P. M.; MATIAS M. J.; MAYER, B.; NUNES, D.; TRANCOSO, V.N. Isotopic and hydrochemical data as indicators of recharge areas, flow paths and water-rock interaction in the Caldas da Rainha-Quinta das Janelas thermomineral carbonate rock aquifer (Central Portugal). Journal of hydrology, v. 476, p. 302-313, 2013. https://doi.org/10.1016/j.jhydrol.2012.10.047

MILLS, G. Luke Howard and the climate of London. Weather, v. 63, n. 6, p. 153-157, 2008.

MOHTADI, M.; LÜCKGE, A.; STEINKE, S.; GROENEVELD, J.; HEBBELN, D.; WESTPHAL, N. Late Pleistocene surface and thermocline conditions of the eastern tropical Indian Ocean. Quaternary Science Reviews, v. 29, n. 7, p. 887-896, 2010. https://doi.org/10.1016/j.quascirev.2009.12.006

NEVES, G., GALLARDO, N., FELÍCIO, R., MACEDO, S., \& VECCHIA, F. Variação da temperatura de superfície em diferentes usos do solo na cidade de São Carlos-SP. GOT, Revista de Geografia e Ordenamento do Território, n. 13, p. 315-336, 2018. http://dx.doi.org/10.17127/got/2018.13.014

OLTRA-CARRIÓ. R.; SOBRINO, J.A.; SÒRIA. G.; BIANCHI, R.; PAGANINI, M. et al. Impact of spatial resolution and satellite overpass time on evaluation of the surface urban heat island effects. Remote Sensing of Environment, v. 117, p. 50-56, 2012. https://doi.org/10.1016/j.rse.2011.04.042

PEREIRA, B. R.; MEES, M. B.; LEITE, M. R.; FILHO, R.M. Análise da gestão ocupacional em Montes Claros-MG: Impactos do uso do solo e suas consequências na temperatura de superfície. Revista Cerrados, v. 15, n. 1, p. 285-311, 2017.

ROUSE JR, J. W. et al. Monitoring vegetation systems in the great Plains with Erts, vol. 351. NASA Special Publication, v.351, p. 309, 1974. 
SAKUNO, N. R. R.; KAWAKUBO, F. S.; SPALEVIC, V.; MINCATO, R. L. Mapeamento de superfícies impermeáveis em áreas urbanas utilizando imagens índices geradas pelo sistema Landsat-5 Thematic Mapper. Revista do Departamento de Geografia, n. spe, p. 190-198, 2017. https://doi.org/10.11606/rdg.v0ispe.132729

SANTOS, R. G.; PRATA-SHIMOMURA, A. R.; CORREIA, E.; FRANCO, M. A. R.; LOPES, A. S. Morfologia Urbana e Corredores de Ventilação como subsídio à Resiliência Urbana. Revista LABVERDE, v. 8, n. 2, p. 12-37, 2017. https://doi.org/10.11606/issn.2179-2275.v8i2p12-37

SOLTANI, A.; SHARIFI, E. Daily variation of urban heat island effect and its correlations to urban greenery: A case study of Adelaide. Frontiers of Architectural Research, v. 6, n. 4, p. 529-538, 2017. https://doi.org/10.1016/j.foar.2017.08.001

SOUSA, L. S.; TRAVASSOS, L. Problemas ambientais urbanos: desafios para a elaboração de políticas públicas integradas. Cadernos Metrópole. n. 19, p.27-47, 2008.

TEIXEIRA, D. C. F.; AMORIM, M. C. D. C. T. Ilhas de calor: representações espaciais de cidades de pequeno porte por meio de modelagem. GEOUSP Espaço e Tempo (Online), v. 21, n. 1, p. 239-256, 2017. https://doi.org/10.11606/issn.2179-0892.geousp.2017.113406

TRINDADE, E. P.; HINNIG, M. P. F.; COSTA, E. M.; MARQUES, J. S.; BASTOS, R.C.; YIGITCANLAR, T. Sustainable development of smart cities: A systematic review of the literature. Journal of Open Innovation: Technology, Market, and Complexity, v. 3, n. 3, p. 11, 2017. https://doi.org/10.1186/s40852-017-0063-2

TUCKER, C. J. Red and photographic infrared linear combinations for monitoring vegetation. Remote sensing of Environment, v. 8, n. 2, p. 127-150, 1979. https://doi.org/10.1016/0034-4257(79)90013-0

USGS - GEOLOGICAL SURVEY UNITED STATES. Explorer Earth. Disponível em: <https://earthexplorer.usgs.gov/>. 2019.

WOO, S.; LITZIUS, K.; KRÜGER B.; IM, M.; CARETTA, L.; RICHTER, K.; MANN, M.; KRONE, A.; REEVE, R. M.; WEIGAND, M.; AGRAWAL, P.; LEMESH I.; MAWASS, M.A.; FISCHER, P.; KLÄUI, M.; BEACH, G. S. D. Observation of room-temperature magnetic skyrmions and their current-driven dynamics in ultrathin metallic ferromagnets. Nature materials, v. 15, n. 5, p. 501, 2016. https://doi.org/10.1038/nmat4593

YANG, Li; QIAN F.; SONG, D.; ZHENG, K. Research on urban heat-island effect. Procedia Engineering, v. 169, p. 11-18, 2016. https://doi.org/10.1016/j.proeng.2016.10.002

ZHA, Y.; GAO, J.; NI, S. Use of normalized difference built-up index in automatically mapping urban areas from TM imagery. International journal of remote sensing, v. 24, n. 3, p. 583-594, 2003. https://doi.org/10.1080/01431160304987

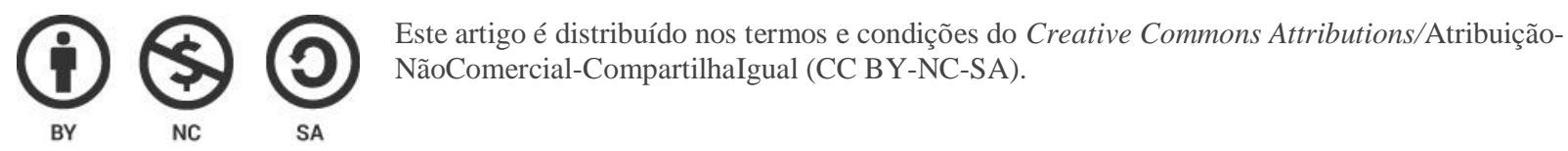

